\title{
Relevant changes in the AJCC 8th edition staging manual for oral cavity cancer and future implications
}

\author{
Luiz Paulo Kowalski, Hugo Fontan Köhler \\ Department of Head and Neck Surgery and Otolaryngology, A C Camargo Cancer Center, São Paulo, Brazil \\ Correspondence to: Luiz Paulo Kowalski, MD, PhD. Department of Head and Neck Surgery and Otolaryngology, A C Camargo Cancer Center, Rua \\ Professor Antônio Prudente 211, São Paulo, Brazil. Email: lp_kowalski@uol.com.br. \\ Comment on: Moeckelmann N, Ebrahimi A, Tou YK, et al. Prognostic implications of the 8th edition American Joint Committee on Cancer (AJCC) \\ staging system in oral cavity squamous cell carcinoma. Oral Oncol 2018;85:82-86.
}

Submitted Mar 01, 2019. Accepted for publication Mar 07, 2019.

doi: $10.21037 /$ cco.2019.03.01

View this article at: http://dx.doi.org/10.21037/cco.2019.03.01

Diagnostic confirmation and staging of cancer are primary steps in cancer patients care. Since its inception, the TNM staging system has as its main focus on an essentially anatomic classification associated with survival endpoints. Ideally, it would allow the stratification of patients in risk groups that have clearly distinct survival outcomes based on anatomic and some nonanatomic variables but similar survival outcomes within each stratum. These strata should be of comparable size and allow prognosis estimation with good approximation for the individual patient and, secondarily, help to define treatment. Not all significant prognostic variables may be used to define the TNM staging. There must be a compromise between practical use and theoretical considerations, with emphasis of use in diverse conditions and reproducibility. A simple system may have a lower accuracy than a complex one but will have a higher compliance by its intended users. The introduction of new variables must take into account these considerations and be practical, reproducible and informative (1). But the changes introduced in the eighth edition mark the greatest update suffered by the TNM staging system for oral cavity cancer (OCC) in the last years.

The initial change in the $8^{\text {th }}$ edition reflect the anatomy of the oral cavity with better definition of the vermillion, lip and oral cavity boundaries. The new transition border between lip and oral cavity rests on the on the division between dry and wet mucosa, with the vermillion staged as skin cancer.

The staging of the primary tumor was primarily affected by the inclusion of the depth of invasion (DOI) alongside tumor extent and invasion of anatomically significant structures. The DOI affects patients that were previously staged as T1 to T3. It replaces the invasion of extrinsic muscles of the tongue which was removed due to the more objective assessment and better hazard discrimination (2).

The addition of DOI aims to improve the staging system of the primary tumor. Initially, tumor thickness, not DOI, was shown to be an important prognostic factor in oral squamous cell carcinoma (OSCC), with a significant higher risk of neck metastasis and worse prognosis for thicker tumors (3). Afterwards, DOI was shown to be an even better predictor than thickness (4). Although different, DOI and tumor thickness may be confused, and the former is the only one included in the new TNM staging for OCC. Tumor thickness is measured as the perpendicular distance from the highest point of the tumor surface to the deepest of the infiltrative front while DOI is measured as the perpendicular distance from the level of the basement membrane to the deepest point of infiltration. But both values show a good correlation with only $5.7 \%$ of patients presenting different stages when tumor thickness is used instead of DOI, suggesting its validity in retrospective studies when the latter is not available (5).

The clinical stage is defined in the pre-operative setting and, theoretically, should be reproducible and correlate with pathological staging. Clinical examination and radiological methods are usually used in conjunction to evaluate OCC. Radiological assessment of the primary site and neck may be performed by ultrasonography, computed tomography (CT) scan and magnetic resonance imaging (MRI) or a combination of these. But pre-operative staging is not as simple as it seems. In a survey of members 
of the American Society of Head and Neck Radiology, only $24.5 \%$ of respondents included the TNM staging in their reports routinely although $49 \%$ considered its inclusion as significant. Major reasons for not inclusion were the lack of clinical data available to the radiologist and legal considerations. For patients with OCC, the lack of definition of axial images regarding superficial mucosal extent was also a major cause of concern (6). The inclusion of DOI and extranodal extension (ENE) may increase the burden on radiologists and decrease the rate of reports with TNM staging.

The use of radiological techniques for evaluating DOI has been the subject of several publications. In a comparison of clinical evaluation and MRI to distinguish DOI above or below 5 millimeters, both methods showed the same sensitivity $(80 \%)$ but the latter had a higher specificity (97\% vs. $84 \%$ ). The positive predictive value of MRI was $89 \%$ compared to $61.5 \%$ of clinical examination (7). The use of contrast-enhanced MRI was evaluated to measure tumor thickness in a prospective series of 13 patients with correlation coefficients of 0.938 and 0.941 , respectively. Both were statistically significant (8).

Intraoral USG has already been subject to multiple evaluations regarding its applicability. A systematic review identified 16 articles on the topic with twelve being selected for metanalysis. It included a total of 464 patients and for 392 patients $(84 \%)$, a correlation between pathological and imaging tumor thickness could be calculated. This correlation was highly significant in 11 studies, the other being a small series of just seven patients. When specific subsites were analyzed, Pearson correlation coefficients of 0.80 for buccal mucosa, 0.88 for oral tongue and 0.90 for floor of mouth were calculated with a tendency for overestimation of tumor thickness. A major drawback of these studies is the focus on tumor thickness, not DOI (9). A significant consideration in its practical use are limitations related to tumor exposure, specially towards the tongue base, and patient toleration of the procedure (10). Another significant issue is which patients benefit from intraoral US. In a German series, early stage patients were more easily assessed than those with bone invasion, limiting their utility to patients with cT1-cT3 patients. But they draw attention to the possibility of intraoperative use for guided resections (11).

Although CT scans are very popular to OCC staging due to accessibility and fast execution, literature reporting its evaluation for DOI is more limited. In patients with primary oral tongue squamous cell carcinoma (SCC), CT scans showed a correlation measured by the Spearman rho of $0.755(\mathrm{P}<0.001)$ with a similar distribution for both over and underestimation of pathological tumor thickness (12). In a retrospective analysis of 354 patients with oral SCC submitted to either CT or MRI, a correlation coefficient of 0.78 was calculated. The time interval between scan and surgery was significant for the final result with patients operated after more than eight weeks having a lower correlation coefficient $\left(\mathrm{R}^{2}=0.62\right)$. Also, interobserver reliability was excellent (0.84). This study is significant for evaluating the concordance between different radiologists while most emphasize the exams were performed by a single examiner (13).

Comparisons between different methods are limited by the small size of most series. A Dutch series comparing US and MRI in 31 patients disclosed better correlation of the former with pathological tumor thickness $\left(\mathrm{R}^{2}=0.87\right.$ vs. $\left.\mathrm{R}^{2}=0.45\right)$. The same series demonstrates difference in the correlation coefficient between a general $(\mathrm{R}=0.05)$ and a dedicated transducer $(\mathrm{R}=0.93)$ although all exams were performed by the same physician (14). Another series evaluates USG and MRI measurements of tumor thickness and correlates these findings with pathological evaluation. The Pearson correlation coefficients were 0.80 for US, 0.69 for T1 postcontrast MRI and 0.64 for T2-weighted MRI (15).

Pathological ENE is a prognostic factor, but it has been assessed by pathologic examination only in patients who underwent neck dissection. ENE must also be evaluated in the preoperative setting. In an analysis of 508 patients with OCC, either CT scans or MRI were compared to pathological staging. In 93 patients, two radiologists had evaluated the exams and interobserver concordance was good (kappa $=0.79$ ). Again, there was a significant correlation between time of exam related to surgery with patients operated after 8 weeks having a worse accuracy. Excluding these patients, CT scan had a higher accuracy than MRI (80\% vs. $63 \%, \mathrm{P}=0.011)$ and when compared to pathological ENE the sensitivity, specificity, positive predictive value and negative predictive value were $52 \%, 96 \%, 93 \%$ and $66 \%$, respectively (16). A direct comparison of the methods in a series of 17 patients showed sensitivity and specificity of $65 \%$ and $93 \%$ for CT scan and $78 \%$ and $86 \%$ for MRI, respectively. But these differences weren't significant for either sensitivity $(\mathrm{P}=0.1317)$ or specificity $(\mathrm{P}=0.3173)$ (17). In respect to ENE, it's important to note that radiological evidence alone may not be enough to upstage a patient. The presence of clinical signs of ENE supported by radiological evidence is necessary for non-pathological ENE diagnosis. The presence of ENE is now mandatory in pathological 
reports to allow for the correct staging and treatment definition. But a formal recommendation in the AJCC manual is the addition of ENE extension to the report. A significant difference in prognosis has been observed according to the extension of ENE (18).

The addition of DOI causes a significant stage migration for patients with oral SCC. In a retrospective series of 298 patients, 22.8\% were upstaged by DOI alone and 29.2\% were upstaged by addition of ENE. Of 62 patients staged as pT1 according to the $7^{\text {th }}$ edition, only $56.5 \%$ remained pT1 in the $8^{\text {th }}$ edition with nine patients restaged as pT3. From the 66 patients staged as pT2, 41 patients $(62.1 \%)$ migrated to pT3 (19). Another external series validated this finding of upstage of the T category after DOI inclusion. It revised 167 patients and found an upstaging of 51 patients (31\%). It allowed for a better discrimination of disease-specific survival than the $7^{\text {th }}$ edition. The number of patients with metastatic neck lymph nodes was small, preventing a stage by stage analysis (20). This change in staging may pose a significant challenge in the decision to perform elective neck dissection in these patients. In our series, we also confirmed a significant stage migration after use of the DOI. Significantly, patients staged as pT1 according to the $7^{\text {th }}$ edition had a rate of occult neck metastasis of $22.4 \%$ that decreased to $11.5 \%$ in patients that remained pT1 after application of the $8^{\text {th }}$ edition criteria. Patients staged as pT2 had a stable incidence of occult neck metastasis in our series. A decision analysis model couldn't demonstrate any benefit of END for pT1 patients in any setting (21). This significant change in occult neck metastasis calls for new clinical trials assessing the need for END in patients staged as $\mathrm{pT} 1$ and $\mathrm{pT} 2$ according to AJCC $8^{\text {th }}$ edition.

In a large series of patients from the National Cancer Database, the introduction of DOI and ENE caused significant changes in staging. Significantly, clinical staging changed in only $1.1 \%$ of patients but there was pathological upstaging in $10.0 \%$ of patients when both editions are compared. There was a survival improvement for patients with clinical stages II, IVa and IVb and patients with pathological stages IVa. Patients with pathological stage IVb had a significant decrease in survival when compared the $7^{\text {th }}$ to $8^{\text {th }}$ editions. In this series, the survival concordance index had no major change when clinical stage is considered (from 0.714 to 0.715 ) and a minor improvement for pathological staging (from 0.699 to 0.704 ). Independent analysis of $\mathrm{T}$ and $\mathrm{N}$ categories showed an inversion of prognosis in $\mathrm{cN} 3 \mathrm{a}$ and $\mathrm{cN} 3 \mathrm{~b}$ with the former having a higher hazard ratio. In the pathological staging, both $\mathrm{T}$ and $\mathrm{N}$ categories showed a hazard increase with progression of staging, except for $\mathrm{pN} 2 \mathrm{~b}$ and $\mathrm{pN} 2 \mathrm{c}$. The conclusion is that in this large retrospective series, the $8^{\text {th }}$ edition offers only a marginal improvement over the $7^{\text {th }}$ edition (22).

The level of neck metastasis was incorporated for primary rhinopharyngeal tumors but not for other primary sites, although it's easy to evaluate and may carry significant prognostic impact. In an analysis of our series, lymph node metastasis in level IV and V were significantly correlated with survival due to an increased risk of distant metastasis (23). Although the $8^{\text {th }}$ edition represents a major change over the previous one and provides better strata separation in most series, no staging system is perfect. There is a need to weight accuracy and predictive power against ease of use and applicability across several different settings (24).

\section{Acknowledgments}

None.

\section{Footnote}

Conflicts of Interest: The authors have no conflicts of interest to declare.

\section{References}

1. Groome PA, Schulze K, Boysen M, et al. A comparison of published head and neck stage groupings in carcinomas of the oral cavity. Head Neck 2001;23:613-24.

2. Lydiatt W, O'Sullivan B, Patel S. Major changes in head and neck staging for 2018. Am Soc Clin Oncol Educ Book 2018;38:505-14.

3. Huang SH, Hwang D, Longwood G, et al. Predictive value of tumor thickness for cervical lymph-node involvement in squamous cell carcinoma of the oral cavity: a metanalysis of reported studies. Cancer 2009;115:1489-97.

4. Shim SJ, Cha J, Koom W, et al. Clinical outcomes for T1$2 \mathrm{~N} 0-1$ oral tongue cancer patients underwent surgery with and without postoperative radiotherapy. Radiat Oncol 2010;5:43-7.

5. Dirven R, Ebrahimi A, Moeckelmann N, et al. Tumor thickness versus depth of invasion - Analysis of the 8th edition American Joint Committee on Cancer Staging for oral cancer. Oral Oncol 2017;74:30-3.

6. Ko B, Parvathaneni U, Hudgins PA, et al. Do Radiologists Report the TNM Staging in Radiology Reports for Head and Neck Cancers? A National Survey Study. AJNR Am J 
Neuroradiol 2016;37:1504-9.

7. Alsaffar HA, Goldstein DP, King EV, et al. Correlation between clinical and MRI assessment of depth of invasion in oral tongue squamous cell carcinoma. J Otolaryngol Head Neck Surg 2016;45:61.

8. Lam P, Au-Yeung KM, Cheng PW, et al. Correlating MRI and Histologic Tumor Thickness in the Assessment of Oral Tongue Cancer. AJR Am J Roentgenol 2004;182:803-8.

9. Klein Nulent TJ, Noorlag R, Van Cann EM, et al. Intraoral ultrasonography to measure tumor thickness of oral cancer: A systematic review and meta-analysis. Oral Oncology 2018;77:29-36.

10. Mark Taylor S, Drover C, Maceachern R, et al. Is preoperative ultrasonography accurate in measuring tumor thickness and predicting the incidence of cervical metastasis in oral cancer? Oral Oncology 2010;46:38-41.

11. Scheer M, Kübler AC, Manawi NN, et al. Endosonographic imaging of tumor thickness in oral squamous cell cancer and its effect on the incidence of lymph node metastases. Mund Kiefer Gesichtschir 2005;9:282-9.

12. Madana J, Laliberté F, Morand GB, et al. Computerized tomography-based tumor-thickness measurement is useful to predict postoperative pathological tumor thickness in oral tongue squamous cell carcinoma. J Otolaryngol Head Neck Surg 2015;44:49.

13. Weimar EAM, Huang SH, Lu L, et al. RadiologicPathologic Correlation of Tumor Thickness and Its Prognostic Importance in Squamous Cell Carcinoma of the Oral Cavity: Implications for the Eighth Edition Tumor, Node, Metastasis Classification. AJNR Am J Neuroradiol 2018;39:1896-902.

14. Lodder WL, Teertstra HJ, Tan IB, et al. Tumour thickness in oral cancer using an intra-oral ultrasound probe. Eur Radiol 2011;21:98-106.

15. Yesuratnam A, Wiesenfeld D, Tsui A, et al. Preoperative evaluation of oral tongue squamous cell carcinoma with intraoral ultrasound and magnetic resonance imagingcomparison with histopathological tumour thickness

Cite this article as: Kowalski LP, Köhler HF. Relevant changes in the AJCC 8th edition staging manual for oral cavity cancer and future implications. Chin Clin Oncol 2019;8(Suppl 1):S18. doi: 10.21037/cco.2019.03.01 and accuracy in guiding patient management. Int $\mathrm{J}$ Oral Maxillofac Surg 2014;43:787-94.

16. Almulla A, Noel CW, Lu L, et al. Radiologic-pathologic correlation of extranodal extension in patients with squamous cell carcinoma of the oral cavity: implications for future editions of the TNM classification. Int J Radiat Oncol Biol Phys 2018;102:698-708.

17. King AD, Tse GM, Yuen EH, et al. Comparison of CT and MRI imaging for the detection of extranodal neoplastic spread in metastatic neck nodes. Eur J Radiol 2004;52:264-70.

18. Brasilino de Carvalho M. Quantitative analysis of the extent of extracapsular invasion and its prognostic significance: a prospective study of 170 cases of carcinoma of the larynx and hypopharynx. Head Neck 1998;20:16-21.

19. Matos LL, Dedivitis RA, Kulcsar MA, et al. External validation of the AJCC Cancer Staging Manual, 8th edition, in an independent cohort of oral cancer patients. Oral Oncol 2017;71:47-53.

20. Tirelli G, Gatto A, Boscolo Nata F, et al. Prognosis of oral cancer: a comparison of the staging systems given in the 7th and 8th editions of the American Joint Committee on Cancer Staging Manual. Br J Oral Maxillofac Surg 2018;56:8-13.

21. Köhler HF, Kowalski LP. A decision analysis model for elective neck dissection in patients with cT1-2 cN0 oral squamous cell carcinoma. Acta ORL Italica. [Epub ahead of print].

22. Cramer JD, Reddy A, Ferris RL, et al. Comparison of the seventh and eighth edition american joint committee on cancer oral cavity staging systems. Laryngoscope 2018;128:2351-60.

23. Köhler HF, Kowalski LP. Prognostic impact of the level of neck metastasis in oral cancer patients. Braz J Otorhinolaryngol 2012;78:15-20.

24. Patel SG, Shah JP. TNM staging of cancers of the head and neck: striving for uniformity among diversity. CA Cancer J Clin 2005;55:242-58. 